\title{
Deformation behaviour of textile electrodes during compression
}

\author{
Diana Germanova-Krasteva ${ }^{1, *}$, and Elena Nikolova ${ }^{2}$ \\ ${ }^{1}$ Technical University of Sofia, Textile Department, 8, K1. Ohridski Blvd, Sofia, Bulgaria \\ ${ }^{2}$ Technical University of Sofia, Department of Electronics, 8, Kl. Ohridski Blvd, Sofia, Bulgaria
}

\begin{abstract}
Textile electrodes are increasingly used to measure biomedical signals (ECG, EMG, EEG) needed for diagnostic activities in medical practice. Tight contact between the electrode and the human body is required to obtain a quality biopotential signal. Pressure is applied to the electrode to secure it. The aim of the work is to study the deformations caused by the applied efforts. The deformation curves of two models of textile electrodes were determined, which have been examined before and after a certain number of washing cycles (10 and 50), in a wide range of pressure changes on the electrodes - from 0.1 to $17 \mathrm{kPa}$. Analyses of the deformation behaviour of the electrodes and its changes after washing are made. Characteristic zones in the deformation curves are defined; reasons for their formation and change after washing are commented.
\end{abstract}

\section{Introduction}

The development of medical science requires the introduction into practice of new materials and technological solutions that improve the practices in the field of long-term medical monitoring.

The modern medical-diagnostic process requires a new approach to the monitoring of biomedical signals. The aim is to minimally disrupt the daily activity and comfort of patients, while maintaining the quality of the obtained bio-stresses, as a basis for good diagnosis, subsequent treatment and rehabilitation of patients [1 - 2].

Leading among the new trends in the field of medical monitoring are the inclusion of intelligent textile systems and materials designed for tracking and recording bio-stresses. The received information is processed and used to select an appropriate diagnostic and treatment-recovery process [3 - 7].

Textile electrodes are designed for installation in systems for long-term registration, storage and communication of biomedical signals. They enter patient monitoring systems due to the following advantages:

- easy to manufacture and implement,

- inexpensive,

- soft,

- do not cause skin irritations and allergic reactions,

\footnotetext{
* Corresponding author: dianakra@tu-sofia.bg
} 
- do not cause discomfort to patients.

The trend is they will gradually replace the existing conventional measurement methods.

Their increasing application requires study of both their electrical characteristics and their mechanical properties [3 - 8]. The reason is that under mechanical impact the electrodes are deformed, and this leads to a change in the electrical characteristics and the information received.

Fig. 1 shows a photograph illustrating the resulting deformations.
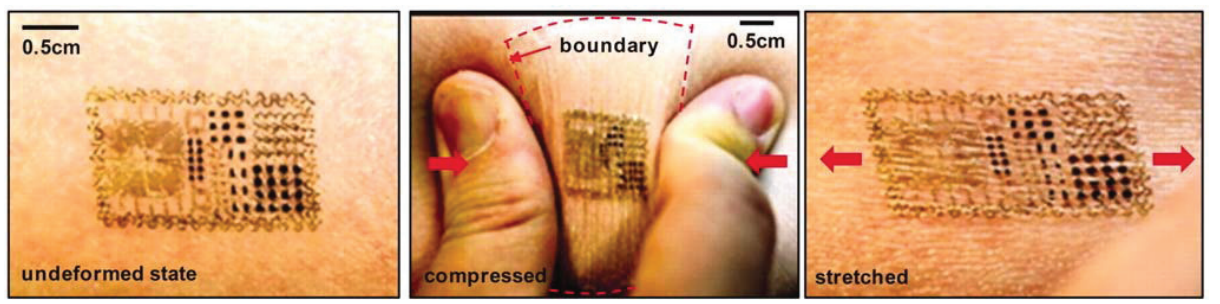

Fig. 1 Multifunctional EEG on skin [3]

Textile electrodes of different shapes, geometries and materials are used to measure biosignals. All these characteristics affect their deformation behaviour and therefore it must be examined for each type of textile electrode separately.

\section{Experimental}

\subsection{Materials}

The deformation behaviour of 2 models of textile electrodes, named "without edging" and "with edging", has been studied.

The "edging-free" model is shown in Fig. 2. It is a round disk with mass $\mathrm{m} \approx 0.7 \mathrm{~g}$, thickness $\delta \approx 4 \mathrm{~mm}$ and diameter $\mathrm{d} \approx 45 \mathrm{~mm}$. Made of polyurethane foam, the surface in contact with the body is covered with a conductive woven fabric of silver threads (Fig. 3 ). On the other hand, the disk is covered with knitted fabric of PA, shown in (Fig. 4).

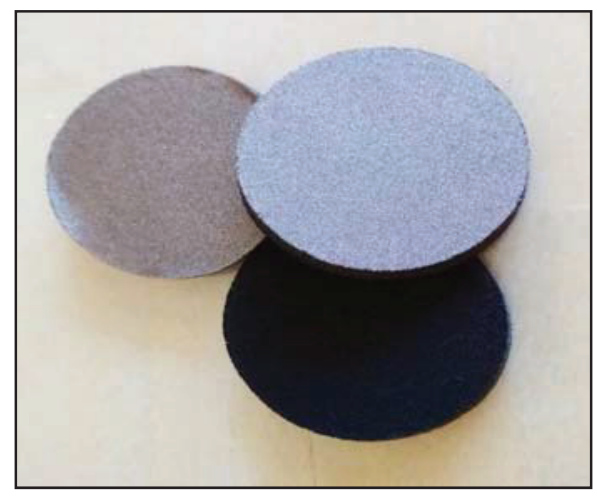

Fig. 2. Textile electrodes without edging.

The "edging" model (Fig. 5) is also made of polyurethane foam, but with much smaller thickness. It is edged on the periphery in order to improve its stability during installation and operation. The diameter is the same as the model without edging, but has a denser 
structure - its mass is about $2.6 \mathrm{~g}$ at a thickness of about $3 \mathrm{~mm}$. On the contact side it is covered with a conductive knitted fabric of silver threads (Fig. 6), and on the other side it is rubberized (Fig. 5). A wiring hole is drilled in the centre of the disc, and the connection is made with a fastening system type "eyelets".

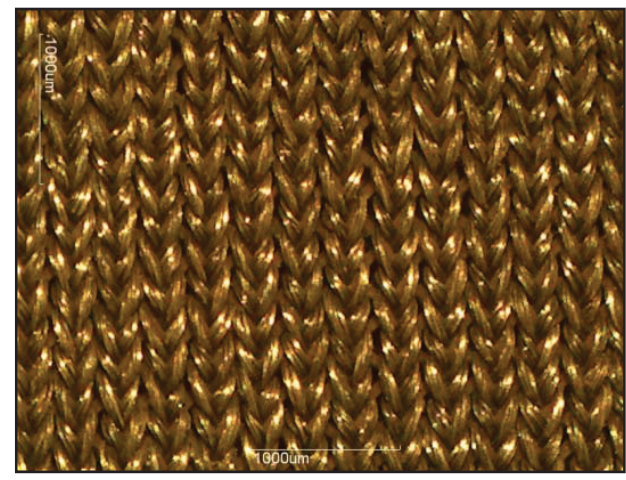

Fig. 3. Contact surface structure (electrodes without edging).

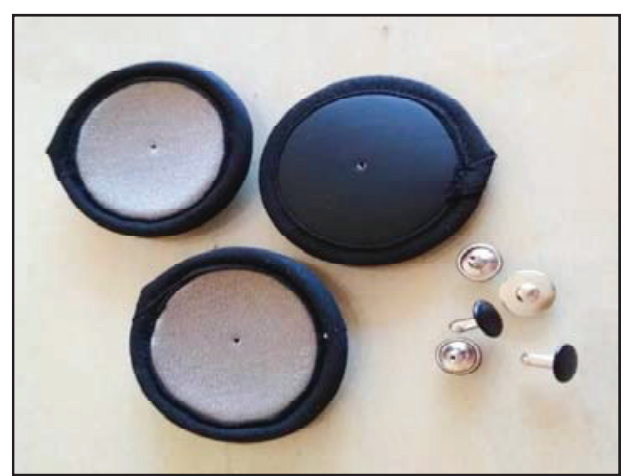

Fig. 5. Textile electrodes with edging.

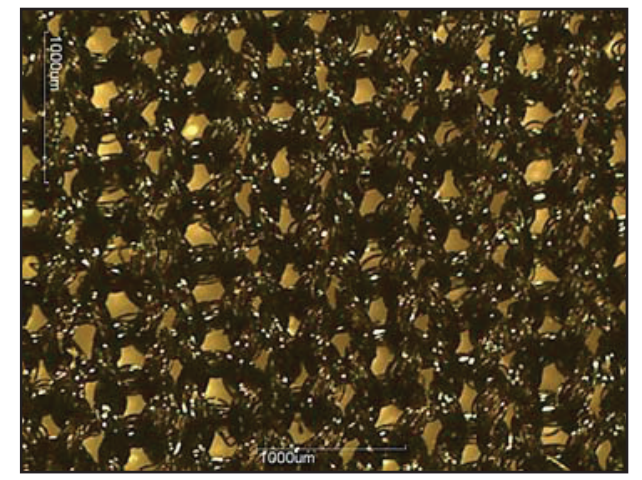

Fig. 4. Non-contact surface structure (electrodes without edging).

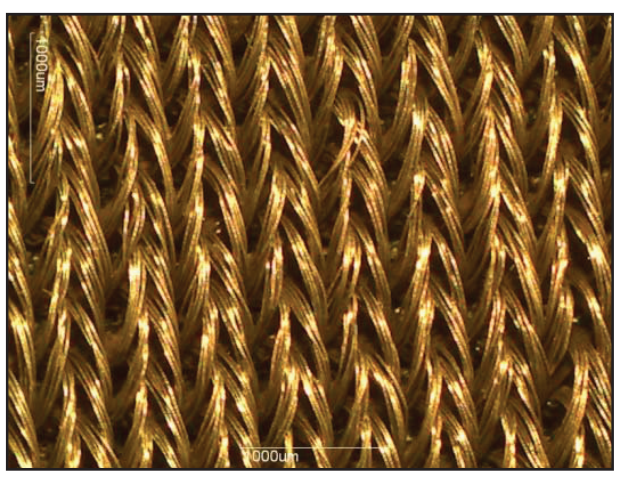

Fig. 6. Structure of the contact surface (electrodes with edging).

All knitted structures are single jersey. Table 1 summarizes the data on the characteristics of knitted structures.

Table 1. Characteristics of knitted structures.

\begin{tabular}{|c|c|c|c|c|}
\hline \multirow{2}{*}{ Electrode model } & \multicolumn{2}{|c|}{ Without edging } & \multirow{2}{*}{ With edging } \\
\cline { 3 - 4 } Characteristic & $\begin{array}{c}\text { Contact } \\
\text { surface }\end{array}$ & $\begin{array}{c}\text { Non-contact } \\
\text { surface }\end{array}$ & \\
\hline \multirow{2}{*}{$\begin{array}{c}\text { Stitch } \\
\text { density }\end{array}$} & courses $/ \mathbf{5} \mathbf{~ c m}$ & 250 & 150 & 130 \\
\cline { 2 - 4 } & wales $/ \mathbf{5} \mathbf{~ c m}$ & 170 & 90 & 100 \\
\hline \multicolumn{2}{|r|}{ Mass per unit area, $\mathbf{g} / \mathbf{m}^{\mathbf{2}}$} & 165 & 94 & N/A \\
\hline
\end{tabular}




\subsection{Methods and devices used}

A digital thickness meter made by Hans Schmidt \& Co GmbH, Germany (Fig. 7) was used to determine the deformation behaviour of the electrodes. In addition to the pressures prescribed in EN ISO 5084: 2002 of $0.1 \mathrm{kPa}$ and $1 \mathrm{kPa}$, the thickness was measured at other reference weights.

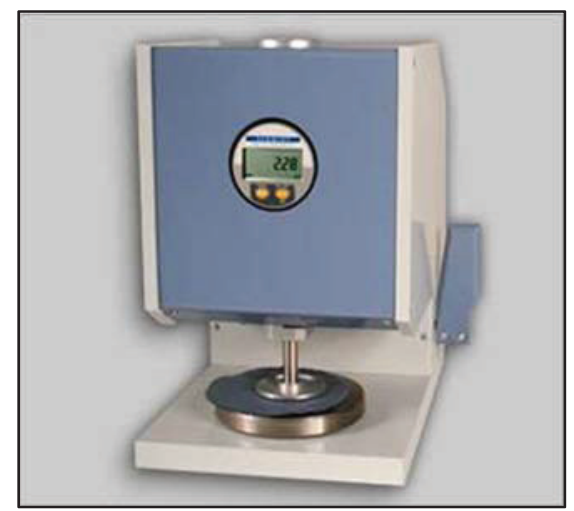

Fig. 7. Digital thickness meter.

\subsection{Results}

In the first stage of the study, the thickness of 10 electrodes from each model was measured, and the data is summarized in Table 2.

The values for the thickness at $0.1 \mathrm{kPa}$ for electrodes with edging are not plotted in it. The reason is that due to the edging the electrodes do not have a flat shape, but a convex shell form. At low values of pressure, it is insufficient for the electrodes to stand up and so in practice not only the thickness but the curvature of the electrode is measured as well. In the last column of Table 2 the average values are calculated.

Table 1. Measured thickness by increasing the pressure.

\begin{tabular}{|c|c|c|c|c|c|c|c|c|c|c|c|}
\hline $\begin{array}{c}\text { Pressure, } \\
\mathbf{k P a}\end{array}$ & $\mathbf{1}$ & $\mathbf{2}$ & $\mathbf{3}$ & $\mathbf{4}$ & $\mathbf{5}$ & $\mathbf{6}$ & $\mathbf{7}$ & $\mathbf{8}$ & $\mathbf{9}$ & $\mathbf{1 0}$ & $\begin{array}{c}\text { Mean } \\
\text { thickness, } \\
\mathbf{m m}\end{array}$ \\
\hline \multicolumn{8}{|c|}{ Electrodes without edging } \\
\hline $\mathbf{0 . 1}$ & 4.37 & 4.34 & 4.37 & 4.35 & 4.31 & 4.31 & 4.27 & 4.23 & 4.20 & 4.23 & 4.30 \\
\hline $\mathbf{1}$ & 4.31 & 4.26 & 4.29 & 4.28 & 4.22 & 4.25 & 4.21 & 4.18 & 4.14 & 4.17 & 4.23 \\
\hline $\mathbf{1 . 5}$ & 4.30 & 4.24 & 4.28 & 4.27 & 4.21 & 4.24 & 4.20 & 4.17 & 4.12 & 4.16 & 4.22 \\
\hline $\mathbf{2}$ & 4.29 & 4.23 & 4.27 & 4.26 & 4.20 & 4.23 & 4.18 & 4.16 & 4.11 & 4.15 & 4.21 \\
\hline $\mathbf{2 . 5}$ & 4.27 & 4.22 & 4.26 & 4.22 & 4.19 & 4.21 & 4.17 & 4.14 & 4.10 & 4.14 & 4.19 \\
\hline $\mathbf{5}$ & 4.25 & 4.20 & 4.24 & 4.17 & 4.16 & 4.19 & 4.15 & 4.12 & 4.07 & 4.10 & 4.17 \\
\hline $\mathbf{1 0}$ & 4.19 & 4.15 & 4.20 & 4.15 & 4.09 & 4.11 & 4.09 & 4.04 & 3.98 & 4.04 & 4.10 \\
\hline $\mathbf{1 5}$ & 2.57 & 2.47 & 2.70 & 2.44 & 2.28 & 2.43 & 2.65 & 2.32 & 2.22 & 2.32 & 2.44 \\
\hline $\mathbf{1 6}$ & 2.44 & 2.30 & 2.52 & 2.28 & 2.15 & 2.25 & 2.48 & 2.16 & 2.07 & 2.15 & 2.28 \\
\hline $\mathbf{1 7}$ & 2.25 & 2.09 & 2.40 & 2.10 & 2.02 & 2.15 & 2.27 & 2.04 & 1.98 & 2.06 & 2.14 \\
\hline
\end{tabular}




\begin{tabular}{|c|c|c|c|c|c|c|c|c|c|c|c|}
\hline \multicolumn{10}{|c|}{ Electrodes with edging } \\
\hline $\mathbf{1}$ & 3.78 & 4.64 & 4.63 & 4.05 & 4.27 & 4.06 & 3.58 & 3.59 & 4.50 & 4.38 & 3.52 \\
\hline $\mathbf{1 . 5}$ & 3.59 & 3.75 & 3.80 & 3.65 & 3.40 & 3.62 & 3.37 & 3.51 & 3.94 & 3.48 & 3.28 \\
\hline $\mathbf{2}$ & 3.44 & 3.46 & 3.54 & 3.42 & 3.23 & 3.44 & 3.25 & 3.38 & 3.60 & 3.25 & 3.17 \\
\hline $\mathbf{2 . 5}$ & 3.34 & 3.34 & 3.43 & 3.32 & 3.15 & 3.35 & 3.19 & 3.26 & 3.50 & 3.10 & 3.10 \\
\hline $\mathbf{5}$ & 3.22 & 3.21 & 3.30 & 3.20 & 3.06 & 3.22 & 3.10 & 3.11 & 3.35 & 2.99 & 3.00 \\
\hline $\mathbf{1 0}$ & 3.03 & 3.05 & 3.09 & 3.05 & 3.02 & 3.02 & 2.99 & 2.94 & 3.14 & 2.87 & 2.84 \\
\hline $\mathbf{1 5}$ & 2.93 & 2.95 & 2.97 & 2.97 & 2.94 & 2.94 & 2.93 & 2.86 & 3.01 & 2.82 & 2.77 \\
\hline $\mathbf{1 6}$ & 2.91 & 2.92 & 2.93 & 2.94 & 2.92 & 2.92 & 2.91 & 2.84 & 2.96 & 2.80 & 2.74 \\
\hline $\mathbf{1 7}$ & 2.87 & 2.90 & 2.92 & 2.91 & 2.90 & 2.90 & 2.90 & 2.82 & 2.94 & 2.78 & 2.70 \\
\hline
\end{tabular}

In Fig. 8 and 9 are shown the deformation curves for the electrodes with and without edging.

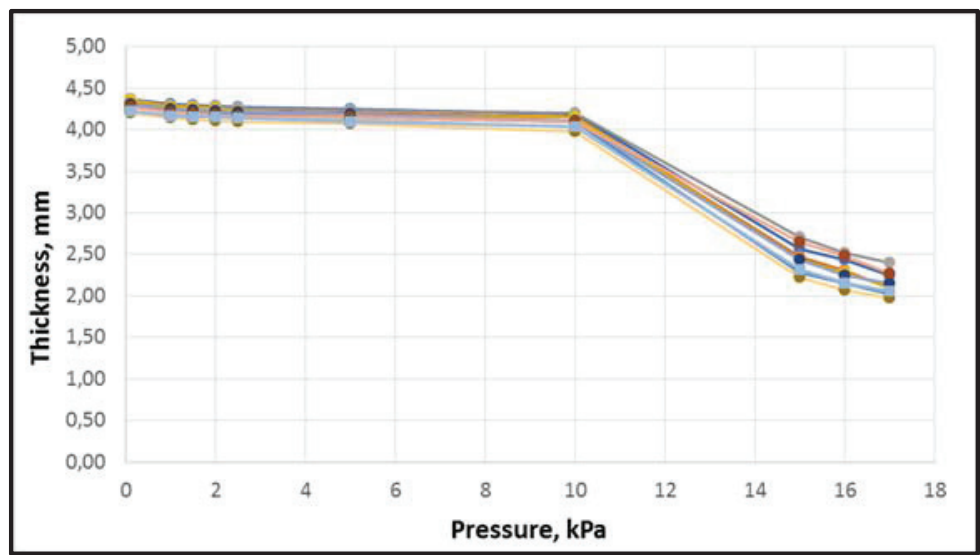

Fig. 8. Deformation curve for textile electrodes without edging.

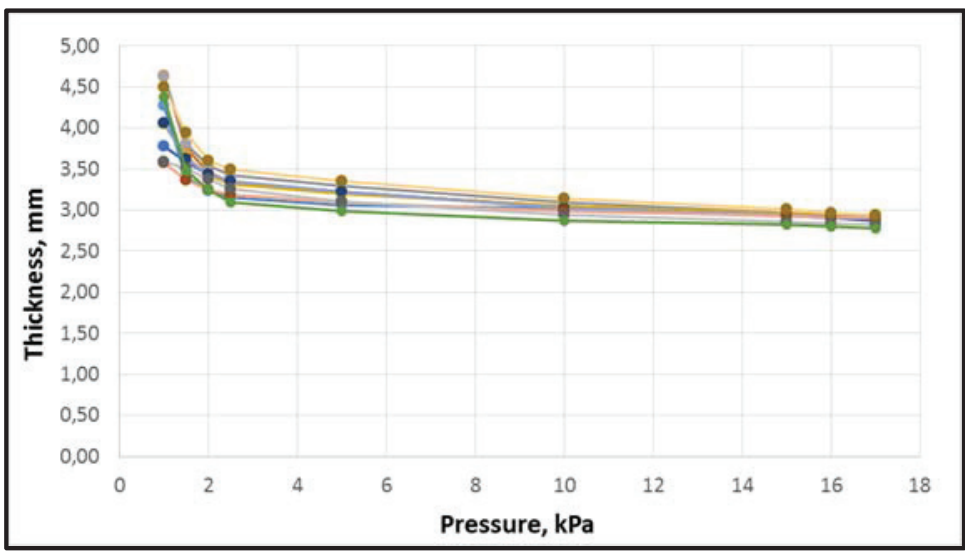

Fig. 9. Deformation curve for textile electrodes with edging.

As shown in Fig. 8 and 9 the deformation curves have a different character due to the different density of the materials.

The textile electrodes without edging have a highly porous structure and deform (compress) more significantly. The curves have three characteristic zones: 1) slight 
deformation (up to $1 \mathrm{kPa}$ ), 2) large deformations at pressure levels from 1 to $14-15 \mathrm{kPa}$ and 3) decrease in deformation gradient (after $15 \mathrm{kPa}$ ). A specific feature of the second stage of the deformation behavior is the gradual reduction of the thickness registered over time. According to the requirements of EN ISO 5084:2002 the thickness reading time is 30 $\mathrm{s}$ - time required for relaxation of the material. While at low and high load levels the deformation proceeds relatively quick - within $3 \div 4 \mathrm{~s}$, for the middle zone there is a change in the thickness during the measurement, and reading is performed after $20 \div 25 \mathrm{~s}$, sometimes $30 \mathrm{~s}$ after the load, i.e. we can talk about strongly expressed viscoelastic behaviour. Some authors have touched on this topic [9 -10].

In the case of textile electrodes with edging, initially a significant change in thickness is observed, but as explained above, it is mainly the result of straightening them and removing / overcoming the curvature. After their final straightening (at a pressure of about $1.5 \mathrm{kPa}$ ) follows a zone (up to $2.5 \mathrm{kPa}$ ) of compression, and finally followed by a smooth, minimal compaction to the end of the examined zone. Here the viscoelastic behaviour is observed at pressures in the range $10-16 \mathrm{kPa}$.

Studies on the degree of compression of the electrodes after $17 \mathrm{kPa}$ have not been done, as even these levels are extremely high to be applied to the human body.

The second stage of the study involves sequential washing of the electrodes according to EN ISO 6330:2012 Textiles - Domestic washing and drying procedures for textile testing. A program for washing coloured fabrics with a water temperature of $40^{\circ} \mathrm{C}$ and centrifugation at $1000 \mathrm{~min}^{-1}$ was used. Liquid detergent with phosphates was used.

The thickness of the two electrode models after 10 and after 50 washing cycles were determined by the same methodology (number of samples and conditions). The aim is to determine whether there is a change in their deformation behaviour as a result of repeated treatment. Similar analyses have been considered [11].

In this case, significant stability of the results and small differences in the values of the individual samples were also found. Therefore, in Table 3 is the summarized data for the average values of the thickness of the new (unwashed) and the washed ones after 10 and 50 cycles of electrodes.

Table 2. Measured thickness by increasing the pressure of new and washed electrodes.

\begin{tabular}{|c|c|c|c|c|c|c|}
\hline \multirow{2}{*}{$\begin{array}{c}\text { Pressure, } \\
\mathbf{k P a}\end{array}$} & \multicolumn{4}{|c|}{ Without edging } & \multicolumn{3}{c|}{ With edging } \\
\cline { 2 - 7 } & New & $\begin{array}{c}\text { After 10 } \\
\text { washing cycles }\end{array}$ & $\begin{array}{c}\text { After 50 } \\
\text { washing cycles }\end{array}$ & New & $\begin{array}{c}\text { After 10 } \\
\text { washing cycles }\end{array}$ & $\begin{array}{c}\text { After 50 } \\
\text { washing cycles }\end{array}$ \\
\hline $\mathbf{0 . 1}$ & 4.30 & 4.41 & 4.44 & - & - & - \\
\hline $\mathbf{1}$ & 4.23 & 4.06 & 4.26 & 4.15 & 3.52 & 4.55 \\
\hline $\mathbf{1 . 5}$ & 4.22 & 4.02 & 4.20 & 3.61 & 3.28 & 4.43 \\
\hline $\mathbf{2}$ & 4.21 & 3.98 & 4.12 & 3.40 & 3.17 & 4.26 \\
\hline $\mathbf{2 . 5}$ & 4.19 & 3.93 & 4.04 & 3.30 & 3.10 & 4.09 \\
\hline $\mathbf{5}$ & 4.17 & 3.73 & 3.82 & 3.18 & 3.00 & 3.82 \\
\hline $\mathbf{1 0}$ & 4.10 & 1.85 & 2.43 & 3.02 & 2.84 & 3.36 \\
\hline $\mathbf{1 5}$ & 2.44 & 1.20 & 1.85 & 2.93 & 2.77 & 3.13 \\
\hline $\mathbf{1 6}$ & 2.28 & 1.11 & 1.70 & 2.91 & 2.74 & 3.06 \\
\hline $\mathbf{1 7}$ & 2.14 & 1.02 & 1.57 & 2.88 & 2.70 & 2.99 \\
\hline $\mathbf{1}$ & 4.30 & 4.41 & 4.44 & 4.15 & 3.52 & 4.55 \\
\hline
\end{tabular}




\begin{tabular}{|c|c|c|c|c|c|c|}
\hline \multirow{2}{*}{$\begin{array}{c}\text { Pressure, } \\
\mathbf{k P a}\end{array}$} & \multicolumn{3}{|c|}{ Without edging } & \multicolumn{3}{c|}{ With edging } \\
\cline { 2 - 7 } & New & $\begin{array}{c}\text { After 10 } \\
\text { washing cycles }\end{array}$ & $\begin{array}{c}\text { After 50 } \\
\text { washing cycles }\end{array}$ & New & $\begin{array}{c}\text { After 10 } \\
\text { washing cycles }\end{array}$ & $\begin{array}{c}\text { After 50 } \\
\text { washing cycles }\end{array}$ \\
\hline $\mathbf{1 . 5}$ & 4.23 & 4.06 & 4.26 & 3.61 & 3.28 & 4.43 \\
\hline $\mathbf{2}$ & 4.22 & 4.02 & 4.20 & 3.40 & 3.17 & 4.26 \\
\hline $\mathbf{2 . 5}$ & 4.21 & 3.98 & 4.12 & 3.30 & 3.10 & 4.09 \\
\hline $\mathbf{5}$ & 4.19 & 3.93 & 4.04 & 3.18 & 3.00 & 3.82 \\
\hline $\mathbf{1 0}$ & 4.17 & 3.73 & 3.82 & 3.02 & 2.84 & 3.36 \\
\hline $\mathbf{1 5}$ & 4.10 & 1.85 & 2.43 & 2.93 & 2.77 & 3.13 \\
\hline $\mathbf{1 6}$ & 2.44 & 1.20 & 1.85 & 2.91 & 2.74 & 3.06 \\
\hline $\mathbf{1 7}$ & 2.28 & 1.11 & 1.70 & 2.88 & 2.70 & 2.99 \\
\hline
\end{tabular}

Graphically, the change in the deformation curves for the two textile electrodes is shown in Fig. 10 and 11.

In the case of electrodes without edging, compression and reduction of the thickness after washing is observed, as well as displacement to the right of the point at which the sharp change of the thickness begins. While for unwashed samples the transition point is at $10 \mathrm{kPa}$, for washed samples it is at $5 \mathrm{kPa}$. After $15 \mathrm{kPa}$ the behaviour is similar, but at clearly lower values of thickness. Research in this area has been done [11-12]

For electrodes with edging after washing there is no change in the type of deformation curves. The pressure level $(2.5 \mathrm{kPa})$ is maintained, at which the deformations become smoother.

It is impressive that in both models of electrodes after the 10th wash there is a decrease in thickness, and after the 50th it is greater. In the case of edging electrodes, the thickness even exceeds that of the new electrodes.

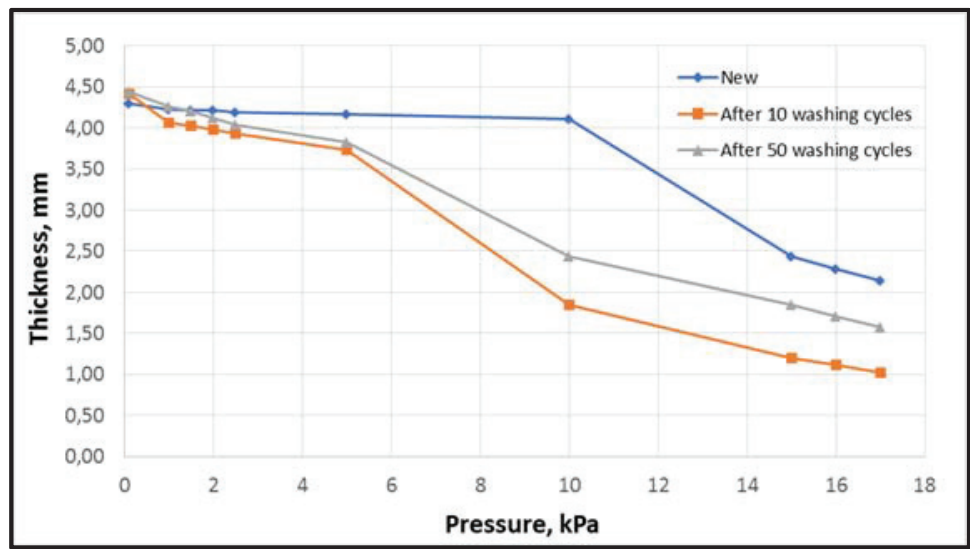

Fig. 10. Deformation curve for textile electrodes without edging - new and washed. 


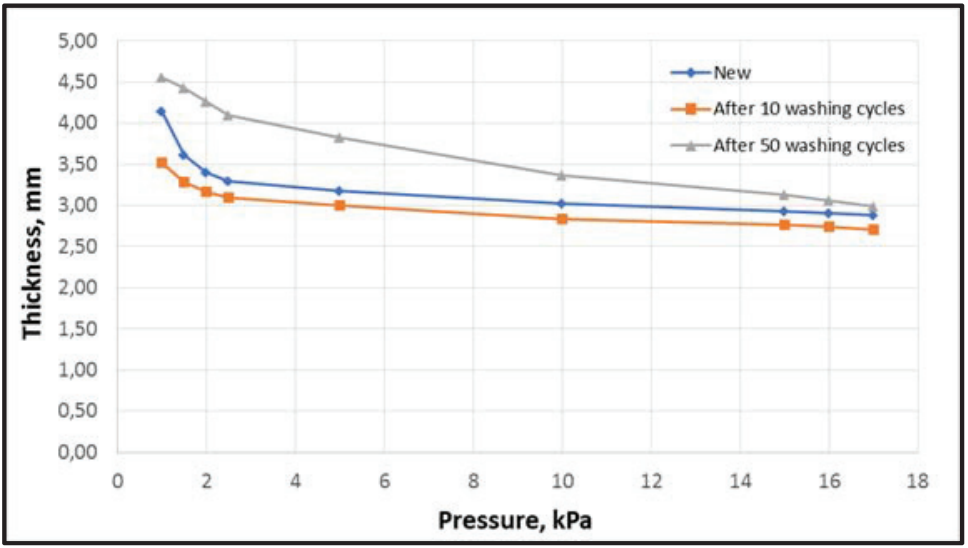

Fig. 11. Deformation curve for textile electrodes with edging - new and washed.

\section{Conclusions}

To the textile electrodes are brought a number of requirements such as abrasion resistance, stain resistance, chemical resistance against secretions such as sweat, and prevention against skin irritation of the wearers.

This article presents the results of a broader study of the textile electrodes application that includes measuring the characteristics of electrical signals and their change after performing different treatments. It identifies and analyses the changes in the deformation behaviour under pressure after cleaning treatment. The reason for conducting this study is the fact that the electrodes will be mounted to a bodysuit made of knitted fabric of cotton yarn and elastomeric thread. This type of products is cleaned mainly by domestic washing. Thus, the conducted research makes it possible to predict the behaviour of the electrodes under real treatment conditions by hygienic maintenance of the product.

A study was carried out for a wide range of variation of compressive forces - from 0.1 to $17 \mathrm{kPa}$.

The presence of characteristic sections of the deformation curves was found, where the polymers have a more pronounced viscous behaviour.

An analysis of the differences in the behaviour of both models of electrodes, as well as the changes that occur after washing is done.

The development is funded by R\&D Sector at TU-Sofia, Project № 202PD0012-02 "Design and products of high-tech textiles".

\section{References}

1. L. Van Langenhove \& all, Smart Textiles for Medicine and Healthcare (2007)

2. I. Iliev \& K. Kostikova, Telemetry of high risk patients with cardiovascular diseases (2014)

3. edited by B. Furht, A. Agarwal, Handbook of Medical and Healthcare Technologies, Springer, 488 (2013)

4. P. Jian \& A. Tiwari, Computers in Biology and Medicine 1-13 (2014)

5. A. Xiang, T. Orathai \& K. George, The Journal of the TI 110(1) (2019)

6. G. Acar \& all, Wearable and Flexible Textile Electrodes for Biopotential Signal Monitoring (2019)

7. H. Mattila, Intelligent textiles and clothing, WPL, 275 (2006) 
8. edited by V. Koncar, Smart Textiles and Their Applications, 718 (2016)

9. A. Cömert, M. Honkala \& J. Hyttinen, BioMedical Engineering OnLine 12(26) (2013)

10. K. Malzahn \& all, Analyst 136 (14) 2912-2917 (2011)

11. edited by I. Jones \& G. K. Stylios Joining Textiles: Principles and Applications, WPL $392(2013)$

12. W. Wuab \& all, FGCS 86, 515-526 (2018) 